\title{
A Rare Cause of Life-Threatening Ketoacidosis: Novel Compound Heterozygous OXCT1 Mutations Causing Succinyl-CoA:3-Ketoacid CoA Transferase Deficiency
}

\author{
Young A Kim ${ }^{1,2}$, Seong Heon Kim ${ }^{1}$, Chong Kun Cheon ${ }^{1}$, and Yoo-Mi Kim ${ }^{3}$ \\ ${ }^{1}$ Department of Pediatrics, Pusan National University Children's Hospital, Yangsan; \\ ${ }^{2}$ Research Institute for Convergence of Biomedical Science and Technology, Pusan National University Yangsan Hospital, Yangsan; \\ ${ }^{3}$ Department of Pediatrics, Chungnam National University Hospital, College of Medicine, Chungnam National University, Daejeon, Korea.
}

\begin{abstract}
Succinyl-CoA:3-ketoacid CoA transferase (SCOT) deficiency is a rare inborn error of ketone body utilization, characterized by episodic or permanent ketosis. SCOT deficiency is caused by mutations in the OXCT1 gene, which is mapped to 5p13 and consists of 17 exons. A 12-month-old girl presented with severe ketoacidosis and was treated with continuous renal replacement therapy. She had two previously unrecognized mild-form episodes of ketoacidosis followed by febrile illness. While high levels of ketone bodies were found in her blood and urine, other laboratory investigations, including serum glucose, were unremarkable. We identified novel compound heterozygous mutations in OXCT1:c.1118T $>\mathrm{G}$ (p.Ile373Ser) and a large deletion ranging from exon 8 to 16 through targeted exome sequencing and microarray analysis. This is the first Korean case of SCOT deficiency caused by novel mutations in $O X C T 1$, resulting in life-threatening ketoacidosis. In patients with unexplained episodic ketosis, or high anion gap metabolic acidosis in infancy, an inherited disorder in ketone body metabolism should be suspected.
\end{abstract}

Key Words: Ketoacidosis, ketone body metabolism, OXCT1

\section{INTRODUCTION}

Ketone bodies can be an alternative energy source if glucose supply is limited, such as during prolonged fasting, or when the body is in a catabolic state. ${ }^{1}$ In patients with defects in ketolysis, the accumulation of ketone bodies can cause fatal ketoacidosis, which may lead to brain damage because of energy depletion that could manifest as psychomotor retardation or even death. ${ }^{1-3}$

Succinyl-CoA:3-ketoacid CoA transferase (SCOT) deficiency (OMIM \#245050) is a rare autosomal recessive inborn error

\footnotetext{
Received: October 4, 2018 Revised: January 2, 2019

Accepted: January 6, 2019

Corresponding author: Yoo-Mi Kim, MD, PhD, Department of Pediatrics, Chungnam National University Hospital, College of Medicine, Chungnam National University, 282 Munwha-ro, Jung-gu, Daejeon 35015, Korea.

Tel: 82-42-280-7288, Fax: 82-42-255-3158, E-mail: ym4805@gmail.com

-The authors have no potential conflicts of interest to disclose.

(C) Copyright: Yonsei University College of Medicine 2019

This is an Open Access article distributed under the terms of the Creative Commons Attribution Non-Commercial License (https://creativecommons.org/licenses/ by-nc/4.0) which permits unrestricted non-commercial use, distribution, and reproduction in any medium, provided the original work is properly cited.
}

of ketone body utilization. ${ }^{1}$ SCOT, which catalyzes the conversion of acetoacetate to acetoacetyl-CoA, is encoded by the OXCT1 gene, mapped to 5p13 and consisting of 17 exons. ${ }^{1,4}$ Affected patients present with intermittent episodes of ketosis preceded by infection or fasting, and usually respond quickly to fluid therapy and bicarbonate infusion. Here, we report the first Korean infant with SCOT deficiency caused by novel compound heterozygous mutations in the OXCT1 gene, who showed life-threatening ketoacidosis requiring continuous renal replacement therapy (CRRT).

\section{CASE REPORT}

A 12-month-old girl presented with tachypnea and vomiting after a one-day fever. Her parents reported symptoms of decreased activity over a period of two days. Born at 40 weeks gestation and weighing $3.18 \mathrm{~kg}$, she was the first child of unrelated healthy parents. The patient was unconscious and was respiring deeply, but otherwise non-specific on physical and neurological examinations. Laboratory results showed high 
Young A Kim, et al.

Table 1. Laboratory Findings for the Patient during Episodes of Ketosis

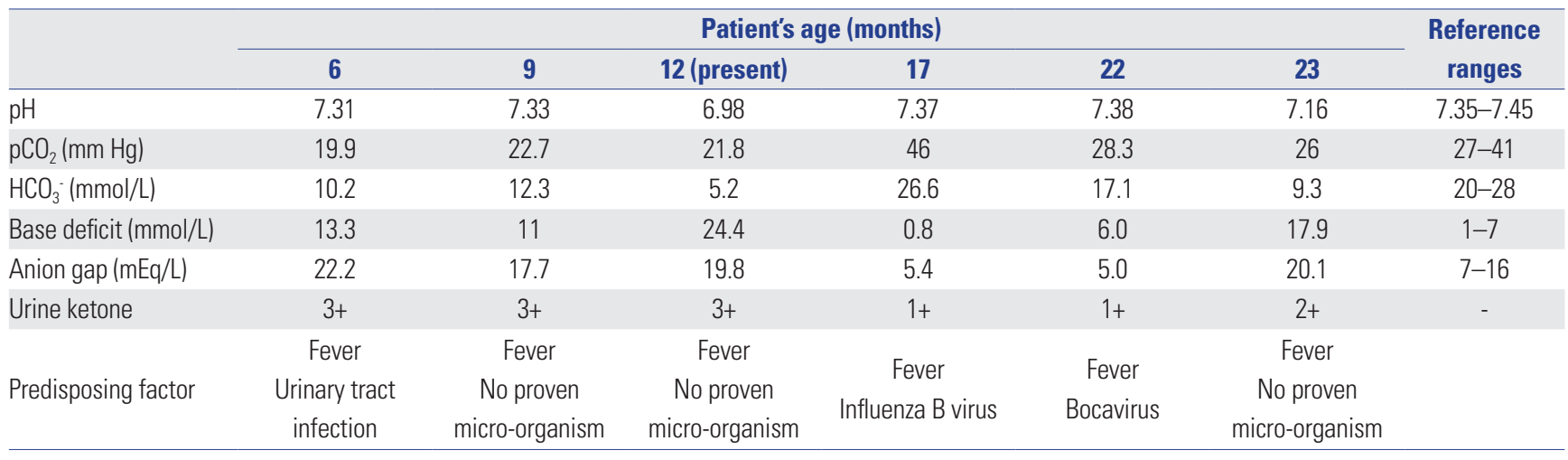

Table 2. Abnormal Laboratory Findings in the Blood and Urine of the Patient

\begin{tabular}{lcc}
\hline & Value & Reference range \\
\hline Blood ketone bodies & & \\
Acetoacetic acid $(\mathrm{mmol} / \mathrm{L})$ & 2.495 & $0.050-0.150$ \\
\hline 3-hydroxybutyrate $(\mathrm{mmol} / \mathrm{L})$ & 0.711 & $0.050-0.300$ \\
Urine organic acid analysis & & \\
\hline Acetoacetic acid $(\mathrm{mmol} / \mathrm{mol} \mathrm{Cr})$ & 5978.1 & $<5$ \\
\hline 3-hydroxybutyrate $(\mathrm{mmol} / \mathrm{mol} \mathrm{Cr})$ & 7751.6 & $<11.4$ \\
\hline 2-hydroxybutyrate $(\mathrm{mmol} / \mathrm{mol} \mathrm{Cr})$ & 261.6 & $<5.1$ \\
\hline Lactic acid $(\mathrm{mmol} / \mathrm{mol} \mathrm{Cr})$ & 570.1 & $<285$ \\
\hline Adipic acid $(\mathrm{mmol} / \mathrm{mol} \mathrm{Cr})$ & 105.3 & $<34.3$ \\
\hline 4-hydroxyphenyllactic acid $(\mathrm{mmol} / \mathrm{mol} \mathrm{Cr})$ & 8.9 & $<3.1$ \\
\hline 4-hydroxyphenylpyruvic acid $(\mathrm{mmol} / \mathrm{mol} \mathrm{Cr})$ & 4.3 & $<0.4$ \\
Plasma carnitine & & \\
\hline Free carnitine $(\mu \mathrm{mol} / \mathrm{L})$ & 12.1 & $27-49$ \\
\hline Acyl carnitine $(\mu \mathrm{mol} / \mathrm{L})$ & 25.3 & $7-19$ \\
\hline Total carnitine $(\mu \mathrm{mol} / \mathrm{L})$ & 37.4 & $38-68$ \\
\hline
\end{tabular}

anion gap metabolic acidosis and ketonuria (Table 1). Serum glucose $(88 \mathrm{mg} / \mathrm{dL})$, ammonia $(50 \mu \mathrm{mol} / \mathrm{L})$, and lactate $(0.5$ $\mathrm{mmol} / \mathrm{L}$ ) levels were normal, and other laboratory findings were also unremarkable. She was admitted to the pediatric intensive care unit for mechanical ventilation, and CRRT was performed because of refractory metabolic acidosis $(\mathrm{pH}$ of 6.94, $\mathrm{HCO}^{-}$of $5.2 \mathrm{mmol} / \mathrm{L}$ ) despite fluid therapy and sodium bicarbonate infusion for 6 hours. CRRT was discontinued after 3 days as ketoacidosis improved, and she was weaned off mechanical ventilation 5 days later.

Her medical records revealed that she had previously been hospitalized twice due to febrile illnesses. Notably, she had metabolic acidosis with ketonuria (Table 1); however, these findings were neglected because her condition rapidly improved after fluid therapy. Given her repeated ketoacidosis episodes in infancy, an inborn error of metabolism was suspected. Although the results of tandem mass spectroscopy and plasma amino acid analysis showed no abnormalities, high levels of ketone bodies were detected in her blood and urine (Table 2). L-carnitine profile showed low levels of free carnitine. These findings were suggestive of a defect in ketone body metabolism, specifically ketone body utilization.

For genetic confirmation, targeted exome sequencing was performed. Initially, the results were interpreted as a novel homozygous mutation: OXCT1:c.1118T>G (p.Ile373Ser). This variant has not been previously reported in SCOT deficiency patients and was not found in gnomAD (http://gnomad. broadinstitute.org/) or the Korean Reference Genome Database (http://coda.nih.go.kr/coda/KRGDB/index.jsp). This novel variant was predicted to be deleterious by SIFT (https:// sift.bii.a-star.edu.sg/), PROVEAN (http://provean.jcvi.org/), and MutationTaster (http://www.mutationtaster.org/), with scores of $0.036,-4.25$, and 0.953 , respectively. However, since this mutation was inherited only from her father and since a loss of heterozygosity was not identified, a deletion in the maternal allele was suspected. Through microarray analysis, a large deletion ranging from exon 8 to 16 in OXCT1 (Fig. 1) was revealed. This large deletion was thought to be inherited maternally; however, microarray analysis was not performed on the patient's mother because the parents wanted to delay the analysis until after they had their next child.

The patient was prescribed a mild protein restricted diet (2.0 $\mathrm{g} / \mathrm{kg} /$ day), with supplements of L-carnitine (100 mg/kg/day), making sure to avoid excessive fat intake. A patient emergency card was given to the patient's parents, and they were instructed to monitor urine ketones using a dipstick test. Ketones were not detected when she was in a stable condition. She experienced three more episodes of ketosis accompanied with febrile illnesses, and ketonuria was reversed quickly by fluid therapy. She has exhibited normal growth and development as of a follow-up at 25 months of age. This study was approved by the Institutional Review Board of Pusan National University Yangsan Hospital (approved number: 05-2018116), and a written informed consent was obtained.

\section{DISCUSSION}

Since the first case of SCOT deficiency was described in 1972, 
and causative pathogenic mutations were found in $O X C T 1$ in 1996, ${ }^{4}$ about 30 patients and 24 different OXCT1 mutations have been reported worldwide..$^{2-13}$ This is the first case of SCOT deficiency in Korea, genetically confirmed with a novel
OXCT1 missense mutation in one allele and a large deletion in the other.

Ketone bodies in the form of acetoacetate and 3-hydroxybutyrate are produced from free fatty acids in the liver. In the

NM_000436.3(OXCT1):c.1118T>G, p.Ile373Ser

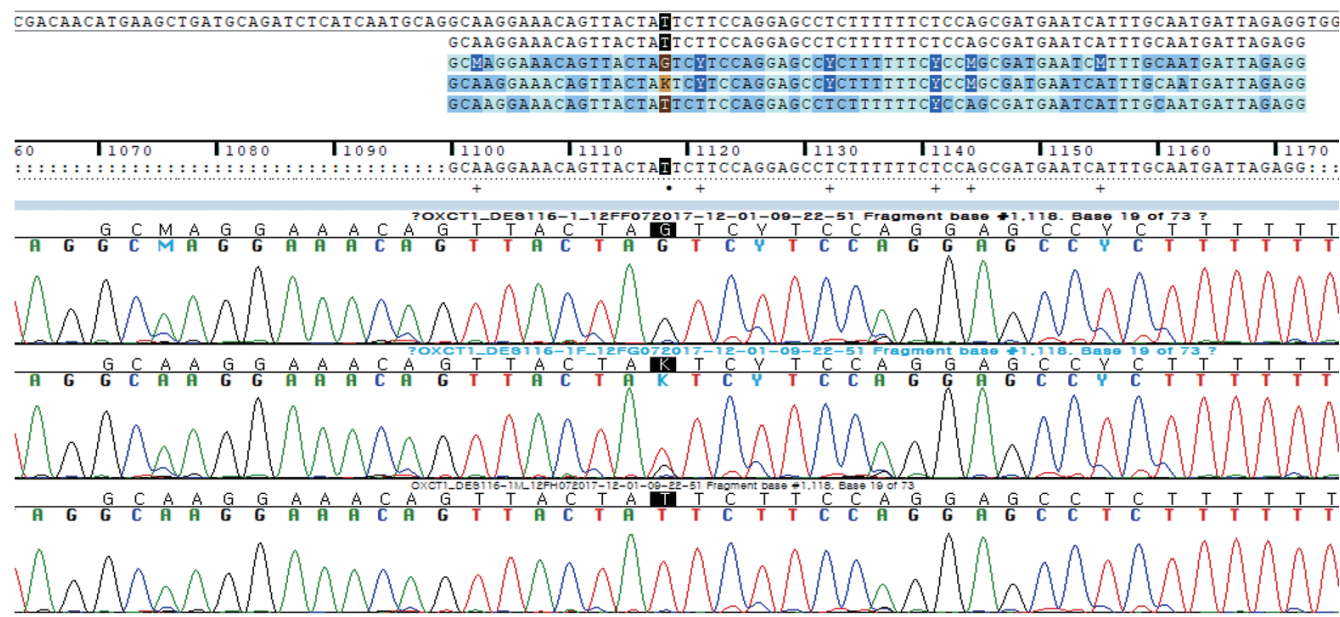

A

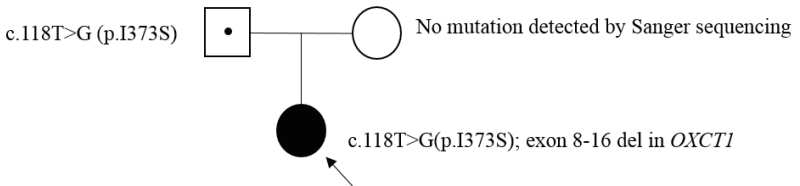

5p13.1(41740874_41838475) deletion array, exon8-16 deletion of OXCT1

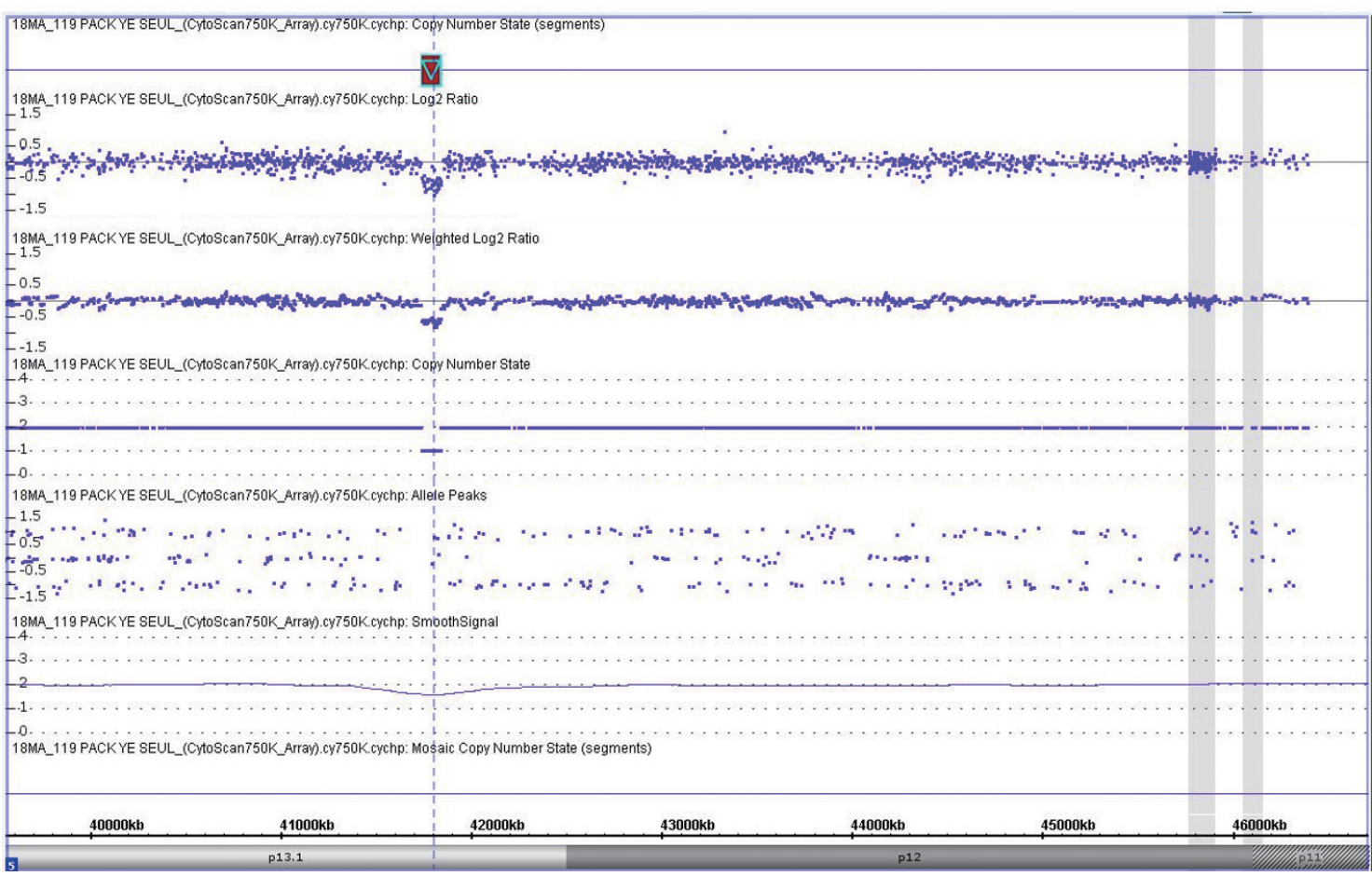

B

Fig. 1. Partial Sanger sequencing of $O X C T 1$ showing a c.1118T $>G$ (p.lle373Ser) mutation in the patient and her father $(A)$, and microarray results for this patient presenting a partial deletion at 5p13.1(41740874_41838475) consistent with the region from exon 8 to 16 of the OXCT1 gene (B). 
mitochondria of extrahepatic tissues, SCOT catalyzes the conversion of acetoacetate to acetoacetyl-CoA, and then, betaketothiolase catalyzes the cleavage of acetoacetyl-CoA into acetyl-CoA, which is an energy source for ATP generation. As SCOT acts in the rate-limiting step in ketolysis, processes downstream of this step do not occur in cases of complete SCOT deficiency, leading to permanent ketosis. ${ }^{1,12}$ Depending on the SCOT enzyme activity they have, patients demonstrate either episodic or permanent ketosis. ${ }^{3,48-12}$ In our patient, episodic ketosis indicated that partial enzyme activity remained, although enzyme activity assays were not performed. Post mortem examination of the first reported SCOT-deficient patient, who died at six months of age, revealed fatty infiltration of the liver and decreased brain size. ${ }^{2}$ However, owing to improved awareness of SCOT deficiency and more experience in the general management of metabolic crises, most patients have a good prognosis, at least those reported in the literature, ${ }^{4-10}$ and cases of neurological impairment and mortality in relation to SCOT deficiency are rarely found. ${ }^{2,3}$

In patients with acute episodes, the recommendation is to treat the metabolic acidosis and infuse sufficient glucose to suppress ketogenesis. When patients are stable, it is recommended that they avoid prolonged fasting and excessive fat intake, mildly restrict protein intake to reduce ketogenesis, and take carnitine supplements as required..$^{12}$ The urine ketone dipstick test, a very simple and inexpensive way to measure ketonuria, is effective for the early detection of ketosis and could prevent a life-threatening crisis.

SCOT deficiency is not detected in newborn screening, and except for high levels of ketone bodies in the blood and urine, patients only have non-specific laboratory results. ${ }^{1}$ Therefore, suspecting a metabolic disorder relating to ketone bodies is the first step in diagnosing SCOT deficiency. Episodic ketosis, ketoacidosis, or high anion gap metabolic acidosis in infancy could be clues for the diagnosis of an inherited disorder of ketone body metabolism.

In summary, we report the first Korean patient with SCOT deficiency with novel compound heterozygous OCXT1 mutations, who showed life-threatening ketoacidosis that was restored by CRRT.

\section{ACKNOWLEDGEMENTS}

We thank the patient and her family for their participation in this study. We wish to thank Green Cross Genome for providing the genetic results in this study.

\section{AUTHOR CONTRIBUTIONS}

Conceptualization: Yoo-Mi Kim. Data curation: Young A Kim. Formal analysis: Young A Kim. Funding acquisition: Young A Kim, Yoo-Mi Kim. Investigation: Yoo-Mi Kim. Methodology: Yoo-Mi Kim. Project administration: Yoo-Mi Kim. Resources: Seong Heon Kim, Chong Kun Cheon. Software: Young A Kim, Yoo-Mi Kim. Supervision: Chong
Kun Cheon, Seong Heon Kim. Validation: Young A Kim, Yoo-Mi Kim. Visualization: Yoo-Mi Kim. Writing_original draft: Young A Kim, Yoo-Mi Kim. Writing_review \& editing: Young A Kim, Seong Heon Kim, Chong Kun Cheon, Yoo-Mi Kim.

\section{ORCID iDs}

Young A Kim https://orcid.org/0000-0002-8332-5200

Seong Heon Kim https://orcid.org/0000-0001-8003-3010

Chong Kun Cheon https://orcid.org/0000-0002-8609-5826

Yoo-Mi Kim https://orcid.org/0000-0002-8440-5069

\section{REFERENCES}

1. Mitchell GA, Fukao T. Inborn errors of ketone body metabolism. In: Scriver C, Beaudet A, Sly W, Valle D, Childs V, Kinzler K, et al., editors. The metabolic and molecular bases of inherited disease. 8th ed. New York (NY): McGraw-Hill, Inc; 2001. p.2327-56.

2. Tildon JT, Cornblath M. Succinyl-CoA:3-ketoacid CoA-transferase deficiency. A cause for ketoacidosis in infancy. J Clin Invest 1972; 51:493-8.

3. Berry GT, Fukao T, Mitchell GA, Mazur A, Ciafre M, Gibson J, et al. Neonatal hypoglycaemia in severe succinyl-CoA:3-oxoacid CoAtransferase deficiency. J Inherit Metab Dis 2001;24:587-95.

4. Kassovska-Bratinova S, Fukao T, Song XQ, Duncan AM, Chen HS, Robert MF, et al. Succinyl CoA:3-oxoacid CoA transferase (SCOT): human cDNA cloning, human chromosomal mapping to $5 \mathrm{p} 13$, and mutation detection in a SCOT-deficient patient. Am J Hum Genet 1996;59:519-28.

5. Shafqat N, Kavanagh KL, Sass JO, Christensen E, Fukao T, Lee $\mathrm{WH}$, et al. A structural mapping of mutations causing succinylCoA:3-ketoacid CoA transferase (SCOT) deficiency. J Inherit Metab Dis 2013;36:983-7.

6. Pretorius CJ, Loy Son GG, Bonnici F, Harley EH. Two siblings with episodic ketoacidosis and decreased activity of succinyl-CoA:3ketoacid CoA-transferase in cultured fibroblasts. J Inherit Metab Dis 1996;19:296-300.

7. Pérez-Cerdá C, Merinero B, Sanz P, Jiménez A, Hernéndez C, Garcáa MJ, et al. A new case of succinyl-CoA: acetoacetate transferase deficiency. J Inherit Metab Dis 1992;15:371-3.

8. Hori T, Yamaguchi S, Shinkaku H, Horikawa R, Shigematsu Y, Takayanagi M, et al. Inborn errors of ketone body utilization. Pediatr Int 2015;57:41-8.

9. Fukao T, Sass JO, Kursula P, Thimm E, Wendel U, Ficicioglu C, et al. Clinical and molecular characterization of five patients with succinyl-CoA:3-ketoacid CoA transferase (SCOT) deficiency. Biochim Biophys Acta 2011;1812:619-24.

10. Longo N, Fukao T, Singh R, Pasquali M, Barrios RG, Kondo N, et al. Succinyl-CoA:3-ketoacid transferase (SCOT) deficiency in a new patient homozygous for an R217X mutation. J Inherit Metab Dis 2004;27:691-2.

11. Barić I, Sarnavka V, Fumić K, Maradin M, Begović D, Ruiter JP, et al. A new case of succinyl-CoA:acetoacetate transferase deficiency: favourable course despite very low residual activity. J Inherit Metab Dis 2001;24:81-2.

12. Fukao T, Mitchell G, Sass JO, Hori T, Orii K, Aoyama Y. Ketone body metabolism and its defects. J Inherit Metab Dis 2014;37:541-51.

13. Sasai H, Aoyama Y, Otsuka H, Abdelkreem E, Naiki Y, Kubota M, et al. Heterozygous carriers of succinyl-CoA:3-oxoacid CoA transferase deficiency can develop severe ketoacidosis. J Inherit Metab Dis 2017;40:845-52. 